\title{
Gynecologic Cancers: Emerging Novel Strategies for Targeting DNA Repair Deficiency
}

\author{
Rebecca S. Kristeleit, MD, PhD, Rowan E. Miller, MD, PhD, and Elise C. Kohn, MD
}

\section{OVERVIEW}

The presence of a BRCA mutation, somatic or germline, is now established as a standard of care for selecting patients with ovarian cancer for treatment with a PARP inhibitor. During the clinical development of the PARP inhibitor class of agents, a subset of women without BRCA mutations were shown to respond to these drugs (termed "BRCAness"). It was hypothesized that other genetic abnormalities causing a homologous recombinant deficiency (HRD) were sensitizing the BRCA wild-type cancers to PARP inhibition. The molecular basis for these other causes of HRD are being defined. They include individual gene defects (e.g., RAD51 mutation, CHEK2 mutation), homozygous somatic loss, and whole genome properties such as genomic scarring. Testing this knowledge is possible when selecting patients to receive molecular therapy targeting DNA repair, not only for patients with ovarian cancer but also endometrial and cervical cancers. The validity of HRD assays and multiple gene sequencing panels to select a broader population of patients for treatment with PARP inhibitor therapy is under evaluation. Other non-HRD targets for exploiting DNA repair defects in gynecologic cancers include mismatch repair (MMR), checkpoint signaling, and nonhomologous end-joining (NHEJ) DNA repair. This article describes recent evidence supporting strategies in addition to BRCA mutation for selecting patients for treatment with PARP inhibitor therapy. Additionally, the challenges and opportunities of exploiting DNA repair pathways other than homologous recombination for molecular therapy in gynecologic cancers is discussed.

O ur increased understanding of cancer biology coupled with increasingly refined technology to examine the cancer genome has offered a rich supply of opportunities applicable to improving outcomes in patients with gynecologic cancer. ${ }^{1}$ We now recognize at least six major interactive pathways involved in DNA damage and repair. ${ }^{2}$ The most recent example of molecularly targeted drug success in patients with ovarian cancer is the development of PARP inhibitors as therapeutics. The PARP inhibitor olaparib was the first drug worldwide to be licensed in 2014 for a molecularly defined population of patients with BRCA-mutated ovarian cancer. The rapidity and success of identifying this molecularly directed and defined therapeutic has catalyzed the gynecologic cancer community to further explore the application of DNA repair inhibitors as a class and question other ways DNA repair defects might be harnessed for novel treatment approaches.

Targeting tumors with defective DNA repair exploits the molecular differences between tumor and normal cells. This mechanism is the basis for tumor-specific cell death induced by PARP inhibitors in patients with BRCA-mutated ovarian cancer. ${ }^{3,4}$ BRCA1 and BRCA2 are essential for maintaining genomic stability through the error-free repair of DNA double-strand breaks via the highly conserved homologousrecombination repair (HRR) pathway. ${ }^{5}$ Although the synthetic lethality between deleterious BRCA mutations and PARP inhibition is well established, ${ }^{4,5}$ it is becoming increasingly evident that gynecologic tumors have other molecular features, germline or somatic, which portend an HRD or BRCA-like susceptibility to platinums and DNA repair inhibitors. Substantial efforts are underway to categorize the breadth of molecular causes of HRD. Individual genetic mutations and whole genome features, expressed as genomic scarring, have been identified as HRD-causing and correlate with potential responsiveness to DNA repair inhibitors. ${ }^{6-9}$ In addition, defects in other DNA repair pathways, such as the MMR pathway (common in patients with endometrial cancer) and cell cycle checkpoint proteins, cause potential vulnerabilities that offer therapeutic possibilities (Fig. 1). ${ }^{10-13}$ Going beyond $B R C A$-targeting in gynecologic cancers highlights the potential for expanded therapeutic strategies. The phenotypic and genotypic consequences of HRD are a particular vulnerability in patients with epithelial ovarian cancer, and new insight shows how other events in endometrial, cervical, and ovarian cancers also may yield a HRD phenotype. An

From the Department of Medical Oncology, University College London Hospital, London, United Kingdom; UCL Cancer Institute, University College London, London, United Kingdom; Clinical Investigations Branch, Cancer Therapy Evaluation Program, National Cancer Institute, Bethesda, MD.

Disclosures of potential conflicts of interest provided by the authors are available with the online article at asco.org/edbook.

Corresponding author: Rebecca S. Kristeleit, MD, PhD, UCL Cancer Institute, 72 Huntley St., London WC1E 6BT United Kingdom; email: r.kristeleit@ucl.ac.uk. 
immediate challenge is learning how best to navigate and apply the new information at both the scientific and clinical levels, where genetically defined treatment decisions are now being made with patients.

\section{HOMOLOGOUS-RECOMBINATION REPAIR AND BRCA1/2}

DNA is constantly subjected to damage by environmental exposures and endogenous activities such as DNA replication and cellular free radical generation. These cause a variety of DNA lesions, including base modifications, double-strand breaks, and single-strand breaks. ${ }^{14}$ DNA repair is critical to maintain genomic integrity by allowing cells to progress through the cell cycle and complete replication without errors. ${ }^{15}$ Homologous-recombination repair is the principle mechanism by which double-strand breaks are repaired. The BRCA1/2 genes, along with other genes in the Fanconi anemia (FA) pathway, encode essential proteins for this process. Homologous-recombination repair is a conservative form of DNA repair that restores the DNA to its original sequence using the homologous normal DNA template during $\mathrm{S}$ and $\mathrm{G} 2 / \mathrm{M}$ phases of the cell cycle. When either BRCA1 or $B R C A 2$ are defective, homologous recombination is dysfunctional and double-strand break repair proceeds using error-prone nonconservative repair mechanisms such as NHEJ and single-strand repair. ${ }^{5}$ Nonhomologous end-joining does not use a DNA template and occurs in G0 or G1, propagating error rather than repairing it. ${ }^{16}$ Any two free DNA break ends are directly ligated during repair of double-strand breaks by NHEJ.

A variety of mechanisms exist for repairing single-strand breaks. These include base excision repair, nucleotide excision repair, and MMR, processes that are modulated by

\section{KEY POINTS}

- The synthetic lethality and validity of using BRCA1/2 mutation as a biomarker predictive of response to PARP inhibition has been confirmed in clinical trials of patients with ovarian cancer.

- Homologous recombinant deficiency (HRD) represents a key vulnerability in patients with high-grade ovarian cancer and possibly other gynecologic cancers, which can be exploited using PARP inhibitors.

- Methods to identify HRD cancers and broaden the applicability of DNA repair inhibitors are being evaluated in the clinic.

- Nonhomologous recombinant DNA repair pathways, such as mismatch repair and nonhomologous end-joining, represent important targets for novel therapeutic strategies in gynecologic cancer.

- Targeting cell cycle checkpoint proteins such as CHK1, CHK2, and WEE-1 that regulate DNA damage and repair is a promising therapeutic approach, particularly in molecular subsets such as $p 53$ mutant and ARID1A-mutated gynecologic cancer.
FIGURE 1. Representation of the Main DNA Repair Pathways and Interaction

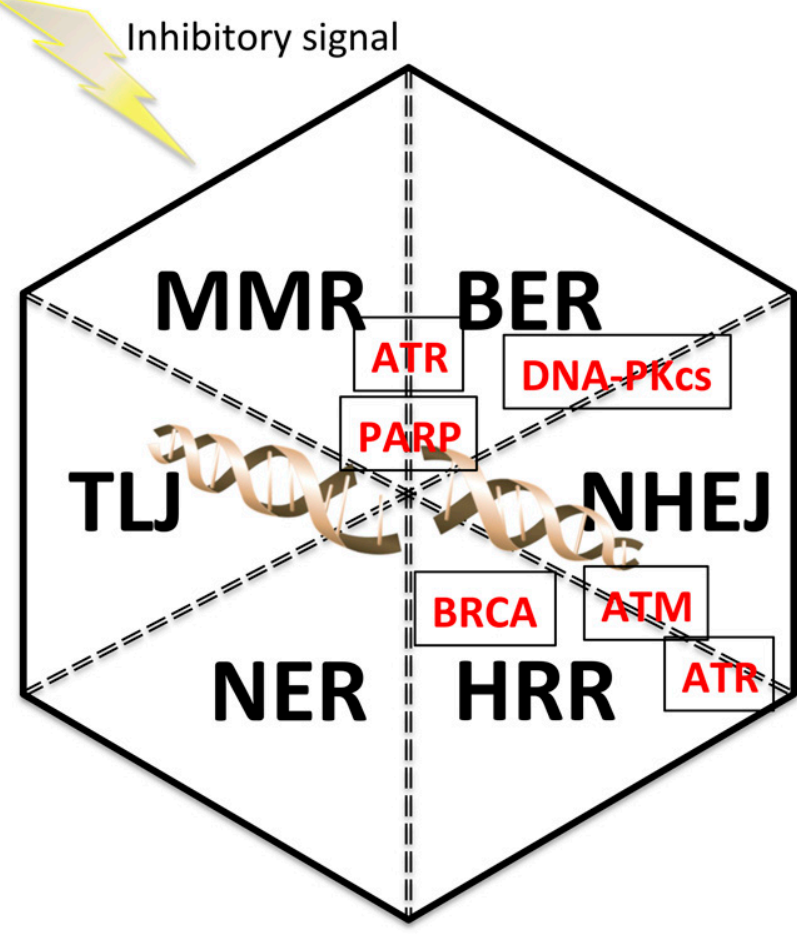

This figure represents the six main DNA repair pathways and their close but discrete interaction. Targetable proteins associated with each pathway for which pharmacological agents exist appear in red.

Abbreviations: MMR, mismatch repair; BER, base excision repair; NHEJ, nonhomologous end-joining; HRR, homologous recombination repair; NER, nucleotide excision repair; TL, translesional joining.

PARP. PARP senses and binds to DNA-break sites, which results in catalytic activation and the recruitment of other components of the DNA repair complex. ${ }^{17}$ If a cell fails to repair single-strand breaks before attempting replication, a double-strand break will then form.

Inherited mutations in the tumor suppressor genes $B R C A 1$ and $B R C A 2$ account for the majority of familial ovarian cancers. ${ }^{18}$ The BRCA1 and BRCA2 protein products function in multiple cellular pathways, including cell cycle regulation and maintenance of genome integrity. ${ }^{19}$ Cells with defective HRR must rely on alternative pathways for DNA repair to survive, thereby providing potential therapeutic targets. Patients with epithelial ovarian cancer and germline or somatic $B R C A 1$ or BRCA2 mutations demonstrate impaired ability to repair double-strand breaks through HRR, which likely explains the increased sensitivity to platinum and the potentially more favorable outcome compared with patients who are wild-type. ${ }^{20,21}$

\section{HOMOLOGOUS-RECOMBINATION REPAIR INHIBITION}

\section{PARP Inhibition}

PARP inhibitors were developed for $B R C A 1 / 2$ mutant epithelial ovarian cancer following observation that $B R C A 1 / 2$ mutations greatly increased the in vitro sensitivity to PARP 
inhibition, exploiting a concept known as synthetic lethality. ${ }^{3,4}$ Synthetic lethality arises when a combination of defects in two or more genes or proteins leads to cell death, whereas a single defect is compatible with cell viability. $B R C A 1 / 2$ defective cells are dependent on non-HR DNA repair and they are sensitive to any induction in doublestrand breaks. PARP inhibition produces stalled replication forks, which increases the number of double-strand breaks and leads to genetic chaos and cell death by apoptosis or senescence. ${ }^{2}$ The "synthetic lethality" between BRCA1/2 mutations and PARP inhibition has been confirmed in clinical trials. ${ }^{22-25}$ Multiple PARP inhibitors, including olaparib (AZD2281), rucaparib (CO-338), veliparib (ABT888), and niraparib (MK4827), are in clinical development either as single agents or in combination therapy for the management of patients with epithelial ovarian cancer (Table 1).

Olaparib, (Lynparza/AZD2281) is the first licensed PARPi for the treatment of BRCA-mutated epithelial ovarian cancer. $^{26,27}$ The initial olaparib phase I study provided clinical proof-of-concept of synthetic lethality between BRCA1/2 mutant tumors and PARP inhibition. Of the expansion phase patients, $40 \%$ attained either RECIST partial or complete response, CA-125 responses by Gynecological Cancer Intergroup criteria, or both. Subsequent phase II studies evaluating olaparib monotherapy in patients with relapsed epithelial ovarian cancer have shown response rates of $31 \%$ to $41 \%$ in BRCA1/2-mutation carriers and up to $21 \%$ in BRCA1/2 wild-type patients. ${ }^{28,29}$ A phase II trial investigating olaparib maintenance therapy following an initial response to platinum therapy showed a progression-free survival (PFS) extension from 4.3 months with placebo to 11.2 months with olaparib (hazard ratio [HR] $0.18 ; 95 \% \mathrm{Cl}, 0.10-0.31$ ) in tumors harboring BRCA1/2 mutations. A benefit for olaparib maintenance in patients with $B R C A$ wild-type tumors was observed, although the magnitude was smaller (7.4 vs. 5.5 months; HR $0.54,95 \% \mathrm{Cl}$, $0.34-0.85) \cdot{ }^{24,30}$ No overall survival (OS) benefit has been observed at the current time of reporting, the reasons for which are likely multifactorial.

\section{Defects in Non-BRCA Homologous-Recombination Repair Genes That Modulate Genomic Stability and May Promote Sensitivity to DNA Repair Inhibitors}

Genomic instability is an important therapeutic target in gynecologic cancers, not just because of the advent of PARPi but also because of the key roles of radiation and platinum therapies in managing them. Platinum analogs induce intraand interstrand purine base cross-links (ICL), which form covalent bonds and stress DNA repair. Repair of ICLs depends on nucleotide excision repair and, secondarily, upon double-strand break formation. ${ }^{14}$ The marked sensitivity of epithelial ovarian cancer to platinum agents is thought to be related to the high frequency of underlying HRR defects. Germline or homozygous somatic mutations in other members of the FA family, such as RAD51C, RAD51D, and $B R I P 1$, increase susceptibility to ovarian cancer. ${ }^{31-33}$ In vitro studies have demonstrated that deficiency in these genes and in other HRR-associated proteins, such as ATM, CHEK1, CHEK2 and CDK12, also confer sensitivity to DNA damage and DNA repair inhibition. ${ }^{31,34-36}$ These have been found in sporadic epithelial ovarian cancer and other cancers in which they appear to function to create a BRCA mutationlike phenocopy. ${ }^{37}$ Understanding other DNA damage mechanisms and potential targets across gynecologic cancers can further extend the success of DNA repair inhibitors, exemplified by PARP inhibitors.

The Cancer Genome Atlas (TCGA) identified mutations now recognized to be related to the HRR pathway in approximately $30 \%$ of high-grade serous ovarian cancers. ${ }^{38}$ This included somatic mutations in BRCA1/2 (3\%), ATM and ATR (2\%), the FANC family (5\%), and hypermethylation of RAD51C (3\%), as well as germline mutations in BRCA1 (9\%) or BRCA2 (8\%). EMSY amplification (13\%), which is proposed to inactive $B R C A 2$, has not been validated in patients yet. Pennington and colleagues used targeted capture and massively parallel genomic sequencing to examine germline and somatic loss-of-function mutations in 30 genes, including 13 HRR genes in 390 epithelial ovarian cancers. ${ }^{36}$ Thirty-one percent of ovarian cancers had a deleterious germline (24\%) and/or somatic (9\%) mutation in one or more of the 13 HRR genes, with similar incidence in serous (31\%) and nonserous ovarian cancers $(28 \%, p=.06)$. The germline or somatic HRR gene mutations predicted platinum sensitivity $(p=.0002)$ and improved OS ( $p=.0006$; Table 2$)$. The majority of germline and somatic HRR gene mutations were in BRCA1/2, and $26 \%$ occurred in other HRR genes. A similar frequency of mutations was observed in patients with nonserous epithelial ovarian cancer but with a different spectrum of targeted genes. The functionality of these additional mutations has been observed with rucaparib in patients with both germline and somatic RAD51C mutations within the ongoing phase II ARIEL2 study (NCT01891344)..$^{39}$ It is now being recognized in epithelial ovarian cancer and in other tumor types that the presence of HRD might be a viable strategy for selection for DNA repair inhibitor trials. For example, $88 \%$ (14 of 16 ) of men with metastatic castration-resistant prostate cancer in the TO-PARP phase II olaparib study with a somatic mutation in an HRR gene, including BRCA1, BRCA2, ATM, the FANC genes, and CHEK2, responded to olaparib compared with only two of $33(6 \%)$ of patients who were wild-type. ${ }^{40}$

\section{New Opportunities Leveraging Genomic Instability}

Recent translational data show BRCA1/2-mutated epithelial ovarian cancer has a greater immune infiltration. ${ }^{41,42}$ It has been suggested that these cancers may have sensitivity to immune checkpoint inhibitors targeting the PD-1/PD-L1 pathway. It is hypothesized that PD-1/PD-L1 targeting agents may preferentially benefit these patients because BRCA1/2mutated and other HRR-deficient tumors have higher numbers of neoantigens. ${ }^{41}$ This hypothesis remains to be tested in clinical trials. 


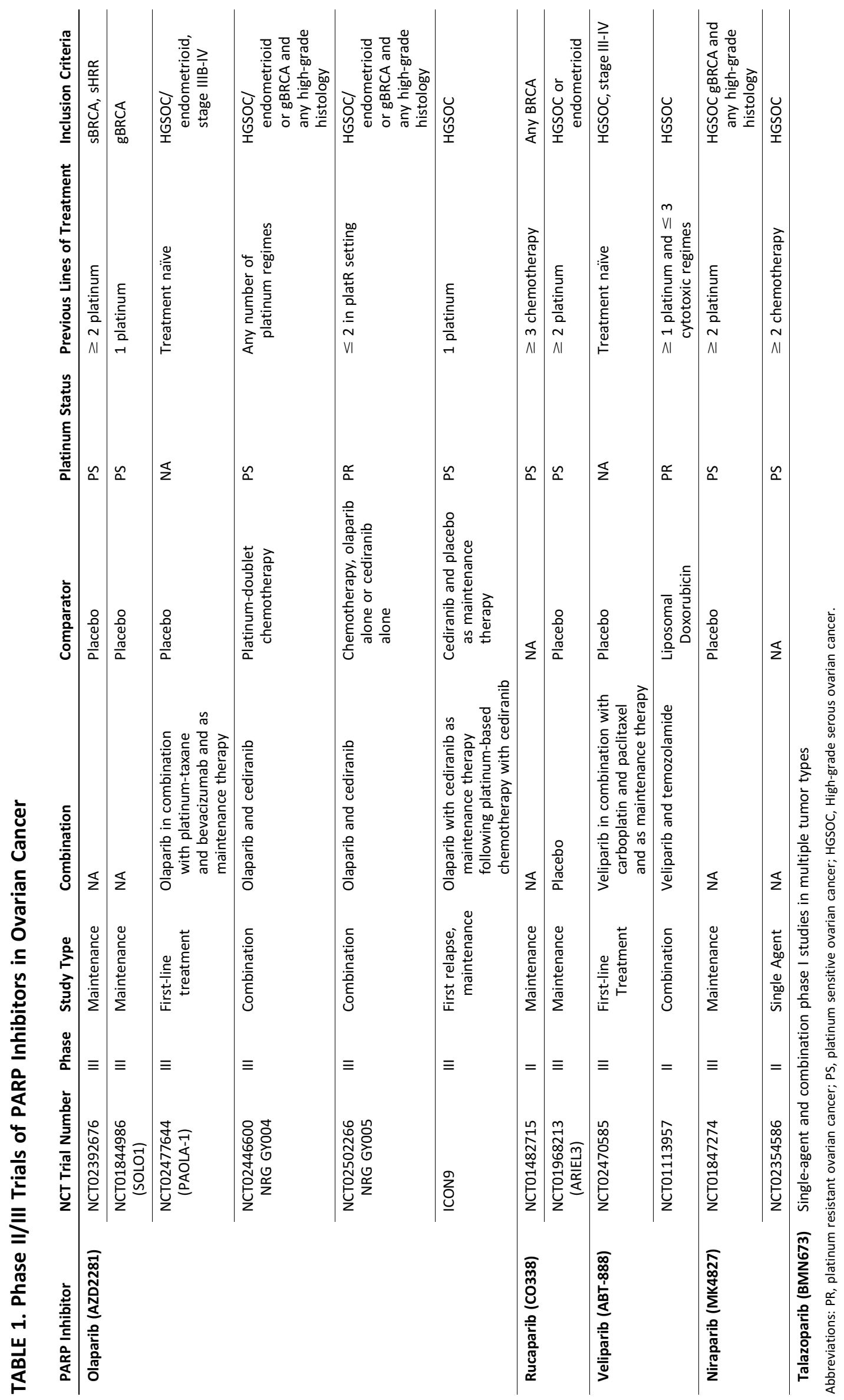


TABLE 2. Known Deleterious Homologous Recombinant Deficiency Gene Frequencies in Ovarian Cancer

\begin{tabular}{|c|c|c|c|}
\hline $\begin{array}{l}\text { HR-Path- } \\
\text { way Gene }\end{array}$ & $\begin{array}{l}\text { Observed Frequency All Epithelial } \\
\text { Ovarian Cancer (\%) }\end{array}$ & $\begin{array}{l}\text { Observed Frequency High-Grade } \\
\text { Ovarian Cancer (\%) }\end{array}$ & References \\
\hline RAD51C & $0.41-2.9$ & 1.9 & $\begin{array}{l}\text { Walsh et } \mathrm{al}^{8} \text {, Pennington et } \mathrm{al}^{36} \text {, Minion et } \mathrm{al}^{83} \text {, Cunningham et } \mathrm{al}^{84} \text {, } \\
\text { Song et } \mathrm{al}^{85}\end{array}$ \\
\hline RAD51D & $0.35-1.1$ & 0.95 & $\begin{array}{l}\text { Pennington et } \mathrm{al}^{36} \text {, Cancer Genome Atlas Research Network }{ }^{38} \text {, Song } \\
\text { et } \mathrm{al}^{85}\end{array}$ \\
\hline RAD51B & 0.06 & 0.95 & Cancer Genome Atlas Research Network ${ }^{38}$, Song et al ${ }^{85}$ \\
\hline RAD50 & $0.2-1.0$ & - & Walsh et $\mathrm{al}^{8}$, Minion et $\mathrm{al}^{83}$ \\
\hline RAD54L & - & 0.5 & Kristeleit et $\mathrm{al}^{86}$ \\
\hline ATM & $0.8-0.86$ & $0.32-1.0$ & $\begin{array}{l}\text { Pennington et } \mathrm{al}^{36} \text {, Cancer Genome Atlas Research Network }{ }^{38} \text {, } \\
\text { Minion et } \mathrm{al}^{83}\end{array}$ \\
\hline BRIP1 & $0.9-4.0$ & $0.32-1.0$ & $\begin{array}{l}\text { Walsh et } \mathrm{al}^{8} \text {, Pennington et } \mathrm{al}^{36} \text {, Cancer Genome Atlas Research } \\
\text { Network }^{38} \text {, Ramus et } \mathrm{al}^{87}\end{array}$ \\
\hline CHEK2 & $0.4-5.0$ & $0.32-1.0$ & $\begin{array}{l}\text { Walsh et } \mathrm{al}^{8} \text {, Pennington et } \mathrm{al}^{36} \text {, Cancer Genome Atlas Research } \\
\text { Network }^{38} \text {, Minion et } \mathrm{al}^{83}\end{array}$ \\
\hline FANCA & - & 0.5 & Kristeleit et $\mathrm{al}^{86}$ \\
\hline FANCI & - & 0.5 & Kristeleit et al $^{86}$ \\
\hline NBN & $0.2-1.0$ & $0.63-1.0$ & $\begin{array}{l}\text { Walsh et } \mathrm{al}^{8} \text {, Pennington et } \mathrm{al}^{36}, \text { Cancer Genome Atlas Research } \\
\text { Network }^{38} \text {, Candido-dos-Reis }\end{array}$ \\
\hline PALB2 & $0.2-2.0$ & 0.63 & $\begin{array}{l}\text { Walsh et } \mathrm{al}^{8} \text {, Pennington et } \mathrm{al}^{36} \text {, Cancer Genome Atlas Research } \\
\text { Network }^{38} \text {, Ramus et } \mathrm{al}^{87}\end{array}$ \\
\hline
\end{tabular}

\section{The Need for Biomarkers: Identifying the Homologous Recombinant Deficiency Signature}

The ability to perform rapid whole-genome sequencing makes it feasible to classify cancers according to their underlying mutational spectrum. A series of in vitro studies has confirmed the presence of large subchromosomal deletions and other genomic changes that cause allelic imbalance and confer an HRD phenotype. ${ }^{6,43,44}$ This has yielded several assays or weighted signatures now being examined as companion diagnostic predictive biomarkers. This HRRdeficient mutational signature or "mutational scar" indicates reliance on error-prone DNA repair pathways. ${ }^{9}$ Array-based technology using single nucleotide polymorphisms genotyping and comparative genomic hybridization has demonstrated that the genomes of high-grade serous ovarian cancer harbor common loss of single parental alleles. Wang and colleagues examined loss of heterozygosity (LOH) and copy number changes in patients with highgrade serous ovarian cancer and divided patients into two clusters of LOH high and LOH low. ${ }^{44}$ High-levels of LOH were associated with platinum sensitivity and improved PFS. $B R C A 1 / 2$-mutant tumors fell within the LOH high group; an increased sensitivity to platinum was seen in the LOH high group even after exclusion of these patients. An LOH-based score has been developed that is strongly associated with functional defects in BRCA1/2 and other genes implicated in the HRR pathway. ${ }^{6}$ Prospective validation of a genomic scar LOH assay is ongoing within the ARIEL2 rucaparib PARP inhibitor phase II trial (NCT01891344) to dichotomize BRCA wild-type patients who benefit from rucaparib. Initial results suggest increased activity for rucaparib within the BRCA-like population with high genomic $\mathrm{LOH}$ compared with the low
LOH population (PFS 7.1 vs. 3.7 months; HR 0.61), although the benefit was not as great as in the BRCA1/2 mutant cohort (PFS 9.4 months). ${ }^{39}$ Additional assays to identify structural changes associated with HRD include the large-scale transitions assay quantifying chromosomal breaks of at least $10 \mathrm{Mb}^{45}$ and the telomeric alleic imbalance score, ${ }^{43}$ both of which correlate with alterations in BRCA1/2 and other HRR pathway genes in patients with ovarian cancer. Although each of these assays offers the exciting prospect of identifying a subset of BRCA-like tumors, which respond to HRR-directed therapy, the relevance of each assay requires prospective validation in clinical trials. As more data emerge from post hoc analyses of tumor samples from completed studies, it is likely that HRD mutational signatures for consideration of DNA repair inhibition therapy will become more established.

\section{DNA REPAIR INHIBITION BEYOND HOMOLOGOUS-RECOMBINATION REPAIR GENES}

Nature has recognized the need to maintain genomic integrity as demonstrated by at least six major-and interactive-DNA repair pathways (Fig. 1). Dissection of HRR has shown this interaction and identified new potential targets for therapeutic intervention. Likewise, dissection of developmental processes in patients with endometrial and colon cancers, and now cervical cancers, has led to recognition of other key DNA repair cancer risk genes. Some of these risk genes are involved in other DNA repair or biologic pathways critical to cellular survival.

Early results implicate deficiency in $A R I D 1 A$, a key component of the chromatin-remodeling complex, as sensitizing 
tumor cells to PARP inhibition in vitro and in animal models. ${ }^{46}$ ARID1A is a suppressor gene. It is recruited by the homologous-recombinant protein ATR, upstream of the cell cycle $\mathrm{G} 2 / \mathrm{M}$ regulator, CHK1, to sites of DNA double-strand breaks where it facilitates efficient processing of doublestrand breaks and sustains DNA damage signaling. Mutations in ARID1A are common in clear cell and endometrioid epithelial ovarian cancer occurring in up to $57 \%$ and $30 \%$ of cases, respectively. ${ }^{47,48}$ Loss of ARID1A expression is also common in patients with endometrioid endometrial cancer (approximately $40 \%{ }^{49}$ ), and similarly in patients with cervical cancer. These results imply that DNA repair inhibitors must be tested more broadly in patients with gynecologic cancers.

DNA repair pathways have cell cycle specificity. ${ }^{50}$ Accordingly, many proteins of the cell cycle, especially within the $\mathrm{G} 2 / \mathrm{M}$ checkpoint, also regulate DNA damage and repair. CHK1/2 and WEE-1 kinases are examples. ${ }^{51-53}$ Agents targeting these kinases have minimal single-agent activity in the absence of p53, or more likely, p53 and second (so far undefined) mutations. Efficacy is much greater when examined in a defined p53 mutant background in which the $\mathrm{G} 1 / \mathrm{S}$ checkpoint is aberrant and also in combination. There is a subset of patients with ovarian cancer with wildtype $B R C A 1 / 2$ function and cyclin $E$ amplification and overexpression. ${ }^{38,54-56}$ Emerging data indicate that blockade of the G1/S checkpoint, such as with pertinent CDK inhibitors, may unmask an unexpected sensitivity to DNA repair inhibitors such as PARP inhibitors. ${ }^{57}$ Furthermore, pharmacologic augmentation of hypoxia, such as in the use of cediranib, can reduce expression of many key homologousrecombinant proteins. This may underpin the activity of the cediranib/olaparib combination in women who have wildtype BRCA. ${ }^{58}$

\section{Targeting Nonhomologous-Recombination Repair DNA Repair Pathways}

Mismatch repair deficiency. Mismatch repair deficiency is a single-strand DNA repair mechanism. It maintains genomic integrity by correcting base substitution mismatches and small insertion-deletion mismatches generated by errors in base pairing during DNA replication. Mismatch repair deficiency is critical to maintaining genomic stability. Failure to recognize and repair DNA mismatches results in microsatellite instability and a mutator phenotype, with mutation rates 100 - to 1,000 -fold higher than in MMR proficient cells. $^{13}$ Loss of one of the MMR proteins (MSH2, MSH6, $\mathrm{MSH} 3, \mathrm{MLH} 1$, and PMS2) is associated with an increased risk of cancers, including endometrial, ovarian, colorectal, and gastric cancers. ${ }^{59}$ Germline mutations in MMR genes give rise to Lynch syndrome, which is associated with a $60 \%$ and $25 \%$ lifetime risk of developing endometrial and colon cancers, and ovarian cancer, respectively. ${ }^{59,60}$ Somatic loss of MMR genes can occur either by mutation or methylation, such that MMR deficiency is associated with up to $30 \%$ of all endometrial cancers. ${ }^{61}$
No direct therapy exists to target MMR-deficient tumors; however, in vitro data suggest a number of potential directions, including inhibition of select DNA polymerases through an accumulation of oxidative DNA damage. Specifically, $M S H 2$ deficiency is synthetically lethal with inhibition of DNA polymerase $\beta$ (POLB), the DNA polymerase that catalyzes nuclear base excision repair. $M L H 1$ deficiency is synthetically lethal with inhibition of DNA polymerase $\gamma$ (POLG), the only polymerase specific to mitochondrial DNA. ${ }^{62}$ Methotrexate has been shown to be lethal to $\mathrm{MSH}$ 2-deficient cells through the accumulation of nuclear oxidative DNA damage, ${ }^{63}$ leading to an ongoing phase II trial in colorectal cancer (NCT00952016). Perhaps more promising is the ability to target secondary mutations that arise as a result of MMR deficiency believed to drive the tumorigenic phenotype. Secondary mutations in the double-strand break DNA repair gene MRE11 are commonly associated with MMRdeficient colorectal cancer and lead to PARP inhibitor sensitivity in vitro. ${ }^{64}$ The role of PARP inhibition in patients with endometrial cancer is ready for assessing in clinical trial with accompanying tissue analysis for microsatellite instability, MRE11, and PTEN status.

The TCGA endometrial cancer group ${ }^{61}$ identified a second subgroup of endometrial cancers characterized by mutations in POLE, a catalytic subunit DNA polymerase epsilon involved in nuclear DNA replication and repair. Mutations in POLE resulted in an ultramutated phenotype with a mutation frequency approximately 10 -fold greater than seen in microsatellite instability tumors. The POLE-mutated subgroup also had extraordinarily good survival. Very preliminary data suggest that the microsatellite instability/POLE phenotype may result in marked increased frequency in neoantigen production, rendering the tumors more immunogenic and, therefore, vulnerable to immunotherapy. ${ }^{65}$ Early preclinical data support the use of anti-PDL1 therapy in POLE-hypermutated and microsatellite instability-positive endometrial cancers because they have greater expression of PD-1, PD-L1, and tumor infiltrating lymphocytes. ${ }^{66}$ Several clinical trials currently underway are evaluating the role of anti-PD-1/PD-L1 therapy in endometrial cancer either as a single agent (e.g., NCT02628067) or in combination with carboplatin and paclitaxel (NCT02549209).

Base excision repair. Many other potential targets have been identified in other DNA repair pathways. The potential therapeutic importance of DNA-PKcs in base excision repair was identified during the demonstration that PARP was not the base excision repair rate-limiting step. ${ }^{16} \mathrm{~A}$ normal role of PARP is to maintain inactive DNA-PKcs, which keeps NHEJ quiet. Agents now in clinical development are targeting DNA-PKcs. DNA-PKcs has been implicated with adverse outcome in patients with epithelial ovarian cancer, suggesting they may be a clinically relevant target in gynecologic cancer. ${ }^{67}$ TRC-102 is an experimental agent targeting base excision repair that also can be considered for patients with gynecologic cancers. 


\section{Cervical Cancer and DNA Repair Defects}

The role of defective DNA repair in cervical cancer is less well established. HPV infection and its associated production of the oncoviral proteins E6 and E7 causes inactivation and degradation of the p53 and pRB tumor-suppressor genes. This results in cell cycle dysfunction and altered DNA repair capacity. ${ }^{68}$ Because of defective DNA damage response, cervical tumor cells are increasingly dependent on residual repair pathways to cope with certain types of DNA damage. In support of this, a correlation between response to DNA damaging therapy and activation of DNA repair pathways has been noted in clinical series, albeit involving relatively small numbers of patients. Patients treated with chemoradiotherapy were found to have high expression of the nucleotide excision repair protein ERCC1 associated with a decreased PFS and worse $O^{69}$ and activation of the $\mathrm{FA} / B R C A$ pathway correlated with treatment failure; conversely, impaired NHEJ repair was related to increased OS in patients treated primarily with radiotherapy. ${ }^{70}$ These observations suggest that therapeutic exploitation of DNA repair pathways may be useful in potentiating chemoradiotherapy, which is the mainstay of treatment for cervical cancer. Numerous early phase trials incorporating modulators of DNA repair such as PARP, ATM and ATR inhibitors, and triapine in combination with standard chemotherapy or radiotherapy currently are underway in patients with advanced cervical cancer (NCT01281852, NCT02223923, NCT02595879, NCT02466971).

\section{NAVIGATING THE NEW INFORMATION}

Picture the cartoon: a patient with the balloon above her head full of question marks. The caption reads "What do I do?" The most difficult question for providers and patients is how to weigh progress and new agents in the context of population data when addressing individual patient decisions. As our understanding of the meaning and roles of HRD mutation and dysfunction progresses, we complicate informing patients.

We always start with what's simple. Germline deleterious $B R C A$ mutation in patients with ovarian cancer is the most common and actionable finding that generally directs simple decisions as described above. BRCA mutation or genomic instability as a result of HRD, in general, allows greater confidence in the use of DNA damaging agents, DNA repair inhibitors, cell cycle inhibitors, and novel combinations that leverage these dysfunctional pathways. We know that BRCAmutation patients with ovarian cancer are more responsive and have better PFS, at least in the first decade after diagnosis. ${ }^{1,71-73}$ Newer data of small, unpublished numbers suggest that any homozygous loss of $B R C A 1 / 2$ may result in a nearly identical phenotype to germline or somatic homozygous loss, an HRD phenotype for which new directions have been outlined.

Germline evaluations of $B R C A$ and other recently identified HRD genes and broader gene panel testing are being done for many patients, often without considering the implications of the potential findings or the treatment plan that the results should inform. ${ }^{74}$ Using a targeted panel of HRR genes may allow the identification of HRD cancers, notwithstanding a number of limitations. With the exception of $B R C A 1 / 2$, each individual HRR gene defect is present at very low frequency in patients with ovarian cancer, and the overall numbers of additional HRD patients identified by testing this way is small (Table 2). ${ }^{8,74,75}$ Homologous recombinant deficiency arises via heterogenous mechanisms, which include epigenetic changes, gene amplifications, and chromosomal translocations. Therefore, a suitable and validated genomic platform is required to capture all patients with potentially actionable HRR aberrations. A third concern is that not all genes are equally important in determining therapeutic response, and the number of variants of uncertain significance is massive, leaving many patients and physicians without guidance.

Addressing whether there is sufficient overall risk to mandate germline testing for all women across all 11 or more genes (panel testing) or to focus on validated biomarker(s) is critical. The latter would allow us to address the more complicated question of the role of haplo-insufficiency complementation in genomic instability of more than one of the homologous-recombinant genes or homologousrecombinant plus another DNA repair pathway gene. Financial, personal, and family costs make navigating this new information more complicated.

\section{Applying the New Information}

BRCA1 or BRCA2 germline or homozygous somatic mutations are now considered predictive biomarkers for platinum- and PARP inhibitor-sensitivity. ${ }^{36} \mathrm{~A}$ woman with a $B R C A 1$ or BRCA2 mutation who progresses on a platinumbased therapy often will respond subsequently. Data are limited to guide application of the platinum-resistant moniker to such women. How to apply the growing data related to complex DNA repair pathways and new agents also is becoming more complex. The first question we need to address urgently in the clinic is how to interpret loss of sensitivity to such agents as the patient moves along the treatment line. Acquisition of secondary mutations in BRCA1 or $B R C A 2$ may result in resumption of $B R C A 1$ or $B R C A 2$ function. ${ }^{76,77}$ Estimates of frequency of these secondary mutations range from single digits to nearly $40 \%$ of cases in the small case numbers examined to date. Other potential molecular events include loss of 53bp1, a regulator of the poor fidelity NHEJ DNA repair pathway, ${ }^{78,79}$ and regulation of phosphoproteins, such as DNA-PKcs, ${ }^{16}$ and others in the DNA repair pathways, such as ATM and ATR. ${ }^{80-82}$ The cause of true platinum resistance in these women is still a conundrum.

\section{WHERE TO GO NEXT?}

DNA repair is a very complex series of parallel and interacting pathways. There are several druggable targets within these pathways. Progress in understanding these pathways and 
TABLE 3. Defining the Decisions?

\begin{tabular}{|c|c|c|}
\hline Event & Implication in Cancer & Opportunities \\
\hline Germline Deleterious gBRCA & $\begin{array}{l}\text { Presumed homozygous deletion, especially breast and } \\
\text { ovarian cancers }\end{array}$ & $\begin{array}{l}\text { DNA damaging agent and PARP inhibitor } \\
\text { susceptibility }\end{array}$ \\
\hline Somatic BRCA Mutation & Role of haplo-insufficiency is unclear & Sensitivity requires homozygous loss of function \\
\hline BRCA1 Promoter Methylation & $\begin{array}{l}\text { Does not appear to confer stable gene downregulation } \\
\text { in ovarian cancer }\end{array}$ & Unclear any benefit \\
\hline BRCA1/2 Downregulation & $\begin{array}{l}\text { Ambient micro-environmental events may affect protein } \\
\text { production or stability }\end{array}$ & $\begin{array}{l}\text { Correlative and preclinical studies suggest this may } \\
\text { yield a sensitive phenotype }\end{array}$ \\
\hline Cyclin E Overexpression & $\begin{array}{l}\text { Cell cycle dysregulation is associated with altered } \\
\text { balance in DNA damage repair }\end{array}$ & $\begin{array}{l}\text { Targeting CDKs alone and in combination with DNA } \\
\text { repair inhibitors }\end{array}$ \\
\hline Unknown and Wild-Type & Work still to do & $\begin{array}{l}\text { Wide-open opportunities for patient care and } \\
\text { learning }\end{array}$ \\
\hline
\end{tabular}

identifying areas of commonality, such as the role of CHK1 in both the G2/M cell cycle checkpoint and downstream of ATM and ATR in DNA repair, has led to new therapeutic approaches. This is an example of the concept that targeting sites of biochemical convergence may yield more than the sum of the inhibition of single independent targets. Chemotherapy is targeted therapy. Most chemotherapies target DNA damage. We now add the therapeutic category of DNA repair inhibitors. How to exploit this new and growing therapeutic category in the context of the treatment of all women with ovarian cancers is a challenge and a great opportunity (Table 3 ). This growth provides great opportunities beyond BRCA.

\section{CONCLUSION AND FUTURE DIRECTIONS}

Targeting defective DNA repair represents a viable treatment option for patients with gynecologic malignancies. Defective HRR is a key vulnerability for patients with highgrade serous epithelial ovarian cancer, which is present in up to $50 \%$ of patients. The use of PARP inhibitors allows the exploitation of molecular differences between tumor normal tissues. To maximize the benefit from PARP inhibition, it is important to identify those tumors not only characterized by $B R C A 1 / 2$ mutations but also those with HRD as a result of other mechanisms. Many questions remain unanswered. These include what determines the best predictor of response to PARP inhibition and how best to use them in the management of epithelial ovarian cancer (i.e., as single agents, in combination with chemotherapy, or as maintenance therapy)? Should we rechallenge with PARP inhibitors for patients in which PARP-directed therapy has failed?

Several trials in progress will likely help answer these questions. It is becoming increasingly apparent that targeting defective DNA repair in patients with nonserous epithelial ovarian cancer, endometrial cancer, and cervical cancer also may have therapeutic potential. This could be achieved either through the identification of non-HRR genes, which may modulate HRR pathways, or by targeting alternative DNA repair pathways such as MMR. Options likely will increase in tandem with our understanding as long as we keep asking relevant questions. The overall aim is, as always, to increase benefit for our patients.

\section{ACKNOWLEDGMENT}

Rebecca Kristeleit, MD, PhD, is supported in part by the UCH/UCL Biomedical Research Centre and UCL Experimental Cancer Medicine Centre.

\section{References}

1. Jayson GC, Kohn EC, Kitchener HC, et al. Ovarian cancer. Lancet. 2014; 384:1376-1388.

2. Dietlein F, Reinhardt HC. Molecular pathways: exploiting tumor-specific molecular defects in DNA repair pathways for precision cancer therapy. Clin Cancer Res. 2014;20:5882-5887.

3. Bryant $\mathrm{HE}$, Schultz $\mathrm{N}$, Thomas HD, et al. Specific killing of BRCA2-deficient tumours with inhibitors of poly(ADP-ribose) polymerase. Nature. 2005; 434:913-917.

4. Farmer $\mathrm{H}, \mathrm{McC}$ abe $\mathrm{N}$, Lord $\mathrm{CJ}$, et al. Targeting the DNA repair defect in BRCA mutant cells as a therapeutic strategy. Nature. 2005;434:917-921.

5. Prakash $R$, Zhang $Y$, Feng $W$, et al. Homologous recombination and human health: the roles of BRCA1, BRCA2, and associated proteins. Cold Spring Harb Perspect Biol. 2015;7:a016600.

6. Abkevich V, Timms KM, Hennessy BT, et al. Patterns of genomic loss of heterozygosity predict homologous recombination repair defects in epithelial ovarian cancer. Br J Cancer. 2012;107:1776-1782.

7. Konstantinopoulos PA, Spentzos D, Karlan BY, et al. Gene expression profile of BRCAness that correlates with responsiveness to chemotherapy and with outcome in patients with epithelial ovarian cancer. J Clin Oncol. 2010;28:3555-3561.

8. Walsh T, Casadei S, Lee MK, et al. Mutations in 12 genes for inherited ovarian, fallopian tube, and peritoneal carcinoma identified by massively parallel sequencing. Proc Natl Acad Sci USA. 2011;108:18032-18037.

9. Watkins JA, Irshad S, Grigoriadis A, et al. Genomic scars as biomarkers of homologous recombination deficiency and drug response in breast and ovarian cancers. Breast Cancer Res. 2014;16:211. 
10. Do K, Doroshow JH, Kummar S. Wee1 kinase as a target for cancer therapy. Cell Cycle. 2013;12:3159-3164.

11. Kobayashi $\mathrm{H}$, Shigetomi $\mathrm{H}$, Yoshimoto $\mathrm{C}$. Checkpoint kinase 1 inhibitors as targeted molecular agents for clear cell carcinoma of the ovary. Oncol Lett. 2015;10:571-576.

12. Krajewska M, Fehrmann RS, Schoonen PM, et al. ATR inhibition preferentially targets homologous recombination-deficient tumor cells. Oncogene. 2015;34:3474-3481.

13. Martin SA, Lord CJ, Ashworth A. Therapeutic targeting of the DNA mismatch repair pathway. Clin Cancer Res. 2010;16:5107-5113.

14. Hoeijmakers JH. Genome maintenance mechanisms for preventing cancer. Nature. 2001;411:366-374.

15. Bouwman P, Jonkers J. The effects of deregulated DNA damage signaling on cancer chemotherapy response and resistance. Nat Rev Cancer. 2012;12:587-598.

16. Patel AG, Sarkaria JN, Kaufmann SH. Nonhomologous end joining drives poly(ADP-ribose) polymerase (PARP) inhibitor lethality in homologous recombination-deficient cells. Proc Natl Acad Sci USA. 2011;108: 3406-3411.

17. Murai J, Huang SY, Das BB, et al. Trapping of PARP1 and PARP2 by clinical PARP inhibitors. Cancer Res. 2012;72:5588-5599.

18. Tutt $A$, Ashworth $A$. The relationship between the roles of BRCA genes in DNA repair and cancer predisposition. Trends Mol Med. 2002;8: 571-576.

19. Gudmundsdottir K, Ashworth A. The roles of BRCA1 and BRCA2 and associated proteins in the maintenance of genomic stability. Oncogene. 2006;25:5864-5874.

20. Cass I, Baldwin RL, Varkey $T$, et al. Improved survival in women with BRCA-associated ovarian carcinoma. Cancer. 2003;97:2187-2195.

21. Tan DSP, Rothermundt $C$, Thomas $K$, et al. "BRCAness" syndrome in ovarian cancer: a case-control study describing the clinical features and outcome of patients with epithelial ovarian cancer associated with BRCA1 and BRCA2 mutations. J Clin Oncol. 2008;26:5530-5536.

22. Fong PC, Boss DS, Yap TA, et al. Inhibition of poly(ADP-ribose) polymerase in tumors from BRCA mutation carriers. N Engl J Med. 2009;361: 123-134.

23. Fong PC, Yap TA, Boss DS, et al. Poly(ADP)-ribose polymerase inhibition: frequent durable responses in BRCA carrier ovarian cancer correlating with platinum-free interval. J Clin Oncol. 2010;28:2512-2519.

24. Ledermann J, Harter $P$, Gourley C, et al. Olaparib maintenance therapy in platinum-sensitive relapsed ovarian cancer. N Eng/ J Med. 2012;366: 1382-1392.

25. Lord CJ, Tutt AN, Ashworth A. Synthetic lethality and cancer therapy: lessons learned from the development of PARP inhibitors. Annu Rev Med. 2015;66:455-470.

26. European Medicines Agency. 2015, EMA Approval of Olaparib. http://www. ema.europa.eu/docs/en_GB/document_library/ EPAR_-_Summary_ for_the_public/human/003726/WC500180153.pdf. Accessed February 1, 2016.

27. Kim $G$, Ison $G$, McKee $A E$, et al. FDA approval summary: olaparib monotherapy in patients with deleterious germline BRCA-mutated advanced ovarian cancer treated with three or more lines of chemotherapy. Clin Cancer Res. 2015;21:4257-4261.

28. Gelmon KA, Tischkowitz $M$, Mackay $H$, et al. Olaparib in patients with recurrent high-grade serous or poorly differentiated ovarian carcinoma or triple-negative breast cancer: a phase 2, multicentre, open-label, non-randomised study. Lancet Oncol. 2011;12:852-861.

29. Kaufman B, Shapira-Frommer R, Schmutzler RK, et al. Olaparib monotherapy in patients with advanced cancer and a germline BRCA1/2 mutation. J Clin Oncol. 2015;33:244-250.

30. Ledermann J, Harter P, Gourley C, et al. Olaparib maintenance therapy in patients with platinum-sensitive relapsed serous ovarian cancer: a preplanned retrospective analysis of outcomes by BRCA status in a randomised phase 2 trial. Lancet Oncol. 2014;15:852-861.

31. Loveday C, Turnbull C, Ramsay E, et al; Breast Cancer Susceptibility Collaboration (UK). Germline mutations in RAD51D confer susceptibility to ovarian cancer. Nat Genet. 2011;43:879-882.

32. Meindl A, Hellebrand $H$, Wiek $C$, et al. Germline mutations in breast and ovarian cancer pedigrees establish RAD51C as a human cancer susceptibility gene. Nat Genet. 2010;42:410-414.

33. Rafnar $T$, Gudbjartsson DF, Sulem $P$, et al. Mutations in BRIP1 confer high risk of ovarian cancer. Nat Genet. 2011;43:1104-1107.

34. Bajrami I, Frankum JR, Konde A, et al. Genome-wide profiling of genetic synthetic lethality identifies CDK12 as a novel determinant of PARP1/2 inhibitor sensitivity. Cancer Res. 2014;74:287-297.

35. McCabe N, Turner NC, Lord CJ, et al. Deficiency in the repair of DNA damage by homologous recombination and sensitivity to poly(ADPribose) polymerase inhibition. Cancer Res. 2006;66:8109-8115.

36. Pennington KP, Walsh $\mathrm{T}$, Harrell $\mathrm{Ml}$, et al. Germline and somatic mutations in homologous recombination genes predict platinum response and survival in ovarian, fallopian tube, and peritoneal carcinomas. Clin Cancer Res. 2014;20:764-775.

37. Lord CJ, Ashworth A. BRCAness revisited. Nat Rev Cancer. 2016;16: 110-120.

38. Cancer Genome Atlas Research Network. Integrated genomic analyses of ovarian carcinoma. Nature. 2011;474:609-615.

39. McNeish IA, Oza AM, Coleman RL, et al. Results of ARIEL2: a phase 2 trial to prospectively identify ovarian cancer patients likely to respond to rucaparib using tumor genetic analysis. J Clin Oncol. 2015;33s (suppl; abstr 5508).

40. Mateo J, Carreira S, Sandhu S, et al. DNA-repair defects and olaparib in metastatic prostate cancer. N Engl J Med. 2015;373:1697-1708.

41. Birkbak NJ, Kochupurakkal B, Izarzugaza JM, et al. Tumor mutation burden forecasts outcome in ovarian cancer with BRCA1 or BRCA2 mutations. PLoS One. 2013;8:e80023.

42. Strickland $\mathrm{K}$, Howitt $\mathrm{BE}$, Rodig SJ, et al. Tumor infiltrating and peritumoral T cells and expression of PD-L1 in BRCA1/2-mutated high grade serous ovarian cancers. J Clin Oncol. 2015;33s (suppl: abstr 5512).

43. Birkbak NJ, Wang ZC, Kim JY, et al. Telomeric allelic imbalance indicates defective DNA repair and sensitivity to DNA-damaging agents. Cancer Discov. 2012;2:366-375.

44. Wang ZC, Birkbak NJ, Culhane AC, et al; Australian Ovarian Cancer Study Group. Profiles of genomic instability in high-grade serous ovarian cancer predict treatment outcome. Clin Cancer Res. 2012;18: 5806-5815.

45. Popova $T$, Manié E, Rieunier $G$, et al. Ploidy and large-scale genomic instability consistently identify basal-like breast carcinomas with BRCA1/2 inactivation. Cancer Res. 2012;72:5454-5462.

46. Shen J, Peng $Y$, Wei $L$, et al. ARID1A deficiency impairs the DNA damage checkpoint and sensitizes cells to PARP inhibitors. Cancer Discov. 2015; 5:752-767.

47. Jones $S$, Wang $T L$, Shih leM, et al. Frequent mutations of chromatin remodeling gene ARID1A in ovarian clear cell carcinoma. Science. 2010; 330:228-231

48. Wiegand KC, Shah SP, Al-Agha OM, et al. ARID1A mutations in endometriosis-associated ovarian carcinomas. N Engl J Med. 2010;363: 1532-1543.

49. Wiegand KC, Lee AF, Al-Agha OM, et al. Loss of BAF250a (ARID1A) is frequent in high-grade endometrial carcinomas. J Pathol. 2011;224: 328-333.

50. Morgan MA, Parsels LA, Maybaum J, et al. Improving the efficacy of chemoradiation with targeted agents. Cancer Discov. 2014;4:280-291.

51. Niida $\mathrm{H}$, Nakanishi M. DNA damage checkpoints in mammals. Mutagenesis. 2006;21:3-9. 
52. Stathis A, Oza A. Targeting Wee1-like protein kinase to treat cancer. Drug News Perspect. 2010;23:425-429.

53. Zhang $Y$, Hunter T. Roles of Chk1 in cell biology and cancer therapy. Int J Cancer. 2014;134:1013-1023.

54. Etemadmoghadam D, Au-Yeung G, Wall M, et al. Resistance to CDK2 inhibitors is associated with selection of polyploid cells in CCNE1amplified ovarian cancer. Clin Cancer Res. 2013;19:5960-5971.

55. Etemadmoghadam D, Weir BA, Au-Yeung G, et al; Australian Ovarian Cancer Study Group. Synthetic lethality between CCNE1 amplification and loss of BRCA1. Proc Natl Acad Sci USA. 2013;110:19489-19494.

56. Karst $A M$, Jones $P M, V e n a ~ N$, et al. Cyclin $E 1$ deregulation occurs early in secretory cell transformation to promote formation of fallopian tube-derived high-grade serous ovarian cancers. Cancer Res. 2014;74: 1141-1152.

57. Alagpulinsa DA, Ayyadevara S, Yaccoby S, et al. A cyclin-dependent kinase inhibitor, dinaciclib, impairs homologous recombination and sensitizes multiple myeloma cells to PARP inhibition. Mol Cancer Ther. 2016;15:241-250.

58. Liu JF, Barry WT, Birrer M, et al. Combination cediranib and olaparib versus olaparib alone for women with recurrent platinum-sensitive ovarian cancer: a randomised phase 2 study. Lancet Oncol. 2014;15: 1207-1214

59. Guillotin D, Martin SA. Exploiting DNA mismatch repair deficiency as a therapeutic strategy. Exp Cell Res. 2014;329:110-115.

60. Aarnio $M$, Mecklin JP, Aaltonen LA, et al. Life-time risk of different cancers in hereditary non-polyposis colorectal cancer (HNPCC) syndrome. Int J Cancer. 1995;64:430-433.

61. Kandoth C, Schultz N, Cherniack AD, et al; Cancer Genome Atlas Research Network. Integrated genomic characterization of endometrial carcinoma. Nature. 2013;497:67-73.

62. Martin SA, McCabe N, Mullarkey M, et al. DNA polymerases as potential therapeutic targets for cancers deficient in the DNA mismatch repair proteins MSH2 or MLH1. Cancer Cell. 2010;17:235-248.

63. Martin SA, McCarthy A, Barber $\mathrm{L}$, et al. Methotrexate induces oxidative DNA damage and is selectively lethal to tumour cells with defects in the DNA mismatch repair gene MSH2. EMBO Mol Med. 2009;1:323-337.

64. Vilar E, Bartnik CM, Stenzel SL, et al. MRE11 deficiency increases sensitivity to poly(ADP-ribose) polymerase inhibition in microsatellite unstable colorectal cancers. Cancer Res. 2011;71:2632-2642.

65. Alexandrov LB, Nik-Zainal S, Wedge DC, et al; Australian Pancreatic Cancer Genome Initiative; ICGC Breast Cancer Consortium; ICGC MMMLSeq Consortium; ICGC PedBrain. Signatures of mutational processes in human cancer. Nature. 2013;500:415-421.

66. Howitt BE, Sholl LM, Ritterhouse L, et al. Association of POLE-mutated and $\mathrm{MSI}$ endometrial cancers with an elevated number of tumorinfiltrating and peritumoral lymphocytes and higher expression of PD-L1. J Clin Oncol. 2015;33s (suppl: abstr 5511).

67. Abdel-Fatah TM, Arora A, Moseley P, et al. ATM, ATR and DNA-PKcs expressions correlate to adverse clinical outcomes in epithelial ovarian cancers. BBA Clin. 2014;2:10-17.

68. Duensing $S$, Münger K. The human papillomavirus type 16 E6 and E7 oncoproteins independently induce numerical and structural chromosome instability. Cancer Res. 2002;62:7075-7082.

69. Balacescu $O$, Balacescu L, Tudoran $O$, et al. Gene expression profiling reveals activation of the FA/BRCA pathway in advanced squamous cervical cancer with intrinsic resistance and therapy failure. $B M C$ Cancer. 2014;14:246.

70. Wilson CR, Davidson SE, Margison GP, et al. Expression of Ku70 correlates with survival in carcinoma of the cervix. Br J Cancer. 2000;83: 1702-1706

71. Candido-dos-Reis FJ, Song H, Goode EL, et al; for EMBRACE; kConFab Investigators; Australian Ovarian Cancer Study Group. Germline mutation in BRCA1 or BRCA2 and ten-year survival for women diagnosed with epithelial ovarian cancer. Clin Cancer Res. 2015;21: 652-657.

72. Jain RK. Normalization of tumor vasculature: an emerging concept in antiangiogenic therapy. Science. 2005;307:58-62.

73. Kotsopoulos J, Rosen B, Fan I, et al. Ten-year survival after epithelial ovarian cancer is not associated with BRCA mutation status. Gynecol Oncol. 2016;140:42-47.

74. Norquist BM, Pennington KP, Agnew KJ, et al. Characteristics of women with ovarian carcinoma who have BRCA1 and BRCA2 mutations not identified by clinical testing. Gynecol Oncol. 2013;128:483-487.

75. Norquist BM, Harrell MI, Brady MF, et al. Inherited mutations in women with ovarian carcinoma. JAMA Oncol. Epub 2015 Dec 30.

76. Edwards SL, Brough R, Lord CJ, et al. Resistance to therapy caused by intragenic deletion in BRCA2. Nature. 2008;451:1111-1115.

77. Norquist B, Wurz KA, Pennil CC, et al. Secondary somatic mutations restoring $B R C A 1 / 2$ predict chemotherapy resistance in hereditary ovarian carcinomas. J Clin Oncol. 2011;29:3008-3015.

78. Bunting SF, Callén $E$, Wong N, et al. 53BP1 inhibits homologous recombination in Brca1-deficient cells by blocking resection of DNA breaks. Cell. 2010;141:243-254.

79. Pennington KP, Wickramanayake A, Norquist BM, et al. 53BP1 expression in sporadic and inherited ovarian carcinoma: relationship to genetic status and clinical outcomes. Gynecol Oncol. 2013;128: 493-499.

80. Guleria A, Chandna S. ATM kinase: much more than a DNA damage responsive protein. DNA Repair (Amst). Epub 2015 Dec 29.

81. Khanna A. DNA damage in cancer therapeutics: a boon or a curse? Cancer Res. 2015;75:2133-2138.

82. Roos WP, Thomas AD, Kaina B. DNA damage and the balance between survival and death in cancer biology. Nat Rev Cancer. 2016;16:20-33.

83. Minion LE, Dolinsky JS, Chase DM, et al. Hereditary predisposition to ovarian cancer, looking beyond BRCA1/BRCA2. Gynecol Oncol. 2015; 137:86-92.

84. Cunningham JM, Cicek MS, Larson NB, et al. Clinical characteristics of ovarian cancer classified by BRCA1, BRCA2, and RAD51C status. Sci Rep. 2014;4:4026.

85. Song $H$, Dicks E, Ramus SJ, et al. Contribution of germline mutations in the RAD51B, RAD51C, and RAD51D genes to ovarian cancer in the population. J Clin Oncol. 2015;33:2901-2907.

86. Kristeleit R, Swisher E, Oza A, et al. Final results of ARIEL2 (Part 1): a phase 2 trial to prospectively identify ovarian cancer $(O C)$ responders to rucaparib using tumor genetic analysis. Eur J Cancer. 2015;51s (suppl; S531).

87. Ramus SJ, Song H, Dicks E, et al; AOCS Study Group; Ovarian Cancer Association Consortium. Germline mutations in the BRIP1, BARD1, PALB2, and NBN genes in women with ovarian cancer. J Nat/ Cancer Inst. 2015;107:11. 